\title{
Alleviation of lateral spillage of two-dimensional hypersonic inlet using waverider-configuration chines
}

\author{
Jian Zhai*, Chen-An Zhang ${ }^{\dagger}{ }^{\ddagger}$, Fa-Min Wang $^{\dagger}$ and Wei-Wei Zhang* \\ * School of Astronautics, Northwestern Polytechnical University, \\ 127 West Youyi Road, Xi'an City, Shaanxi Province 710072, P. R. China \\ $\dagger$ Institute of Mechanics, Chinese Academy of Sciences, \\ No. 15 Beisihuanxi Road, Beijing 100190, P. R. China \\ $\ddagger_{z h c h \_a @ i m e c h . a c . c n}$
}

Received 15 September 2019

Accepted 15 February 2020

Published 4 June 2020

\begin{abstract}
Hypersonic inlet is an important part of the propulsion system of hypersonic airbreathing vehicles. However, the performance of the two-dimensional hypersonic inlet, a major type of hypersonic inlets, is considerably deteriorated for lateral spillage. In this study, waverider-configuration chines mounted on the lateral sides of a two-dimensional three-staged external-compression hypersonic inlet for a Mach number of 6.0 are investigated to determine their ability to alleviate the lateral spillage. The chines are built by using a waverider design method. The numerical results suggest that a severe flow spillage induced by three-dimensional effect shows up near the lateral edge of the inlet without chines, which degrades the mass-flow ratio and flow uniformity. In contrast, the waverider-configuration chines effectively alleviate the lateral spillage. Consequently, the mass-flow ratio and the flow uniformity are both improved significantly.
\end{abstract}

Keywords: Hypersonic inlet; lateral spillage; waverider design method; streamline tracing technology; mass-flow ratio.

PACS number: 47.40.ki

\section{Introduction}

Hypersonic air-breathing vehicles (HAVs), which can fly at more than Mach number of five in the atmosphere, ${ }^{1}$ have received much attention in recent years. Compared with subsonic or supersonic vehicles, hypersonic vehicles have many potential advantages, including (1) dramatically reducing the traveling time ${ }^{2} ;(2)$ used as reusable launch vehicles $(\mathrm{RLVs}) . \underline{3}$ To date, many countries have conducted researches on HAV projects.

$\ddagger$ Corresponding author. 
An important subsystem of an HAV is the inlet which is used to capture and compress air mass-flow. A major type of them is a two-dimensional inlet. This kind of inlet is easy to design, and its geometry is variable for wide-speedrange flight. However, lateral spillage, induced by three-dimensional effect, may significantly degrade its performance, including flow uniformity and mass-flow ratio. Two methodologies, sidewall ${ }^{4,5}$ and lateral chine,,$\frac{6,7}{}$ have been used to restrain the lateral spillage. The control mechanism is reducing the cross-flow in the flow field. The inlets with sidewalls can capture almost all the free-stream; but the shock waves and boundary layers, induced by sidewalls, may degrade the uniformity of flow filed ${ }^{5}$ and there are no benefits for improving the aerodynamic performance of the vehicle. By contrast, mounting lateral chines on the forebody, $\frac{6}{}$ do not bring out undesired shock waves and boundary layers, and well-designed chines may improve the aerodynamic performance. However, experimental results show that only one-half of the surface streamlines entered into the inlet which indicates existence of significant lateral spillage. $\frac{7}{}$

Fortunately, in the flow-field of a waverider, the cross-flow is very small. $\frac{8}{}$ Therefore, the waverider-configuration chines are developed to alleviate the lateral spillage of a two-dimensional hypersonic inlet with three flat ramps in this study. It is found that the lateral spillage is alleviated significantly after mounted the chines.

\section{Design Methodology}

Figure 1 shows a sketch of the model. According to the Osculating Flow field Method, $\underline{8}$ a waverider design method, the procedure used to generate the model begins by assuming the upper surface geometry and the shock wave geometry on the base plane, which are labeled as blue dash curves in Fig. 1(a). Osculating planes are defined to be normal to the local shock wave geometry. $\bar{A}$ basic flow, shown in Fig. 1(b), is then imposed on each osculating plane. The leading edge is subsequently calculated. The lower surface is constructed by tracing streamlines from the leading edge, while only the streamlines behind the shock wave of Ramp 1 in basic flow are used. The upper surface is constructed by tracing from the leading edge in the freestream direction. Figure 2 shows the three-view of the model with chines.

Incidentally, the basic flow is a solution of the compressible Euler equations at a freestream Mach number of 6.0. The configuration used to calculate the basic flow

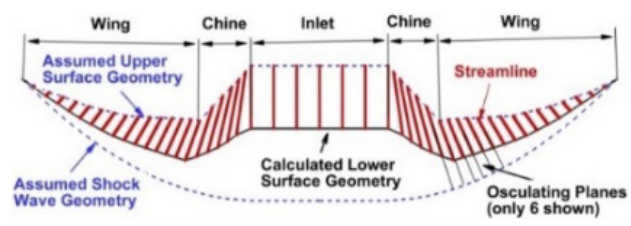

(a) Base plane

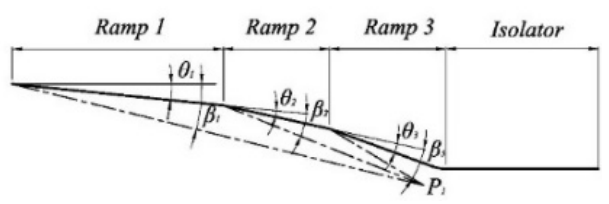

(b) Basic flow

Fig. 1. (Color online) Sketch of the inlet with chines. 


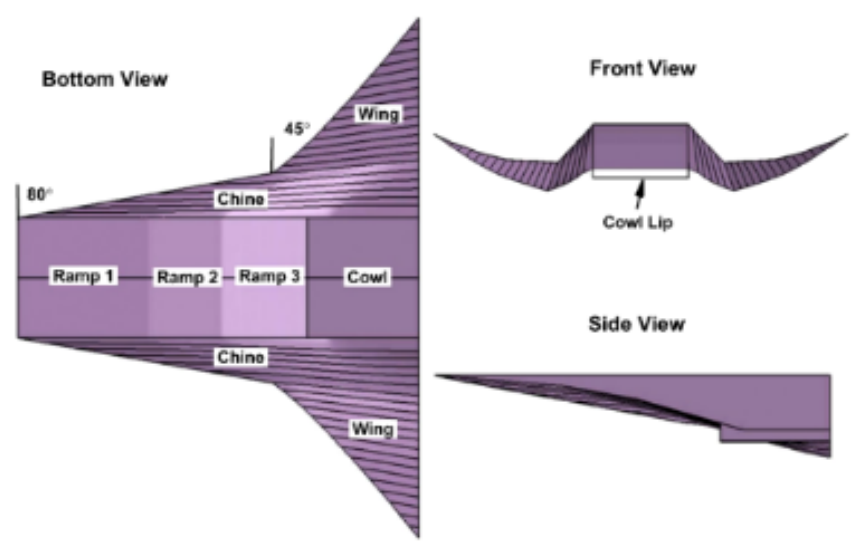

Fig. 2. (Color online) Three-view of the model with chines.

is constructed by three ramps and an isolator. The shock waves, plotted as dash lines in Fig. 1(b), intersect at point P1. The deflection angle $(\theta)$ and the wave angle $(\beta)$ of each ramp are defined by the Oswatitsch principle, $\frac{9}{}$ the total deflection angle of the inlet is $16^{\circ}$.

\section{Numerical Method}

The numerical study is carried out using the compressible Reynolds-averaged Navier-Stokes (RANS) equations. The no-slip, adiabatic wall boundary conditions (BCs) are imposed on all surfaces of the models with and without chines. The freestream BC is imposed on far-field. The freestream Mach number is 6.0. The static pressure is $1197 \mathrm{~Pa}$ and temperature is $226.5 \mathrm{~K}$. The supersonic extrapolation outflow BCs are imposed on external outlet and the isolator exit. The symmetry $\mathrm{BC}$ is imposed on the symmetry plane.

The unstructured grid of the model without chine contains 0.95 million nodes, whereas the other one contains 1.39 million nodes. Grids are refined near the wall, leading edge, ramp corners, lateral edge of the inlet and cowl lip.

The effectiveness of the numerical method is verified by comparing it with the experimental results. $\frac{10}{}$ The comparative results suggest the numerical and experimental results agree well. The numerical errors of the fine and standard grids are $0.42 \%$ and $0.98 \%$, respectively. For saving computational resources, the standard grid (1.39 million nodes) is used for numerical simulation.

\section{Results and Discussion}

Figure 3 shows the flow field of the model without chines. The dimensionless crossflow velocities shown in Fig. 3(a), labeled as $V_{z} / V_{\infty}$, are blanked above 0.15 for better clarity. The results suggest a severe lateral spillage, induced by three-dimensional effect, showing up near the lateral edge. It indicates that the mass-flow ratio is 


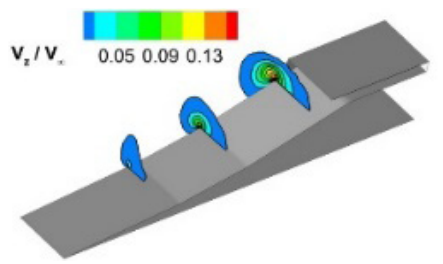

(a) cross-flow velocity

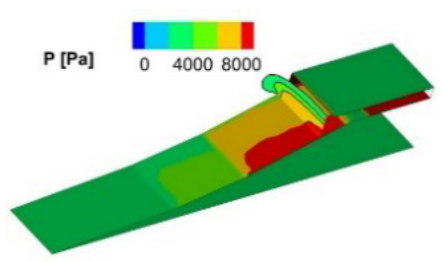

(b) Pressure contours

Fig. 3. (Color online) Flow field of the model without chines.

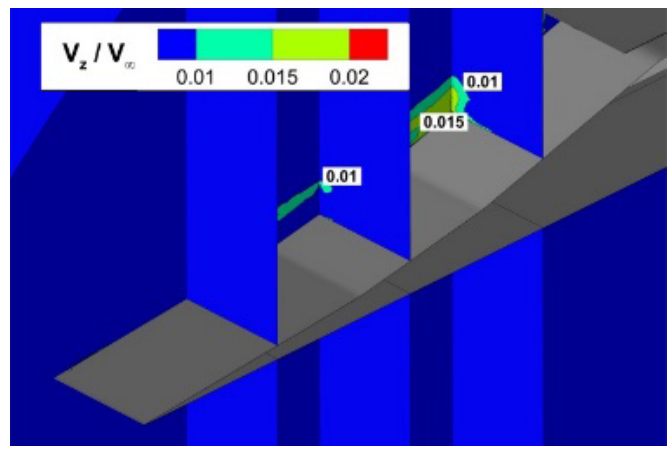

Fig. 4. (Color online) Dimensionless cross-flow velocity of model with chines.

decreased. Figure 3(b) shows the pressure contours on the model surfaces and a slice near the cowl-lip. The results suggest that the pressure near the lateral edge is much smaller than that near the symmetry plane. It indicates that the flow uniformity is also degraded.

Figure 4 shows the dimensionless cross-flow velocity of the model with chines. These results suggest that the dimensionless cross-flow velocity becomes much smaller than that of the model without chines. This indicates that the lateral spillage is alleviated by the chines. The mass-flow ratios $\phi$ and averaged total pressure recovery $\sigma$ at the isolator exit are shown in Table 1 . The results suggest that after mounted the chines, $\phi$ and $\sigma$ increase by $25.0 \%$ and $3.2 \%$, respectively. It indicates that the control strategy using waverider-configuration chines to alleviate the lateral spillage of two-dimensional hypersonic inlet is effective. The inlet performance is significantly improved as compared to that of the model without chines.

Table 1. Comparison of inlet performances at isolator exit.

\begin{tabular}{llcr}
\hline & Model without chines & Model with chines & Error \\
\hline$\phi$ & 0.78 & 0.975 & $25.0 \%$ \\
$\sigma$ & 0.378 & 0.39 & $3.2 \%$ \\
\hline
\end{tabular}




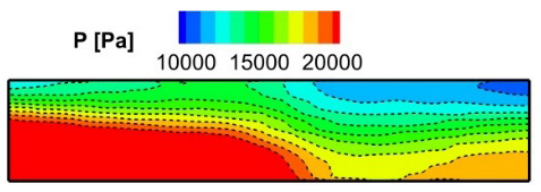

(a) Model without chines



(b) Model with chines

Fig. 5. (Color online) Pressure contours at the isolator exit.

Figure 5 shows the pressure contours at the isolator exit. The results suggest that the flow uniformity of the model with chines is much better than that of the model without chines. It indicates that the flow uniformity of the inlet is significantly improved by adding the waverider-configuration chines.

\section{References}

1. J. D. Anderson, Fundamentals of Aerodynamics (Tata McGraw-Hill Education, New York, 2010).

2. H. Taguchi et al., Acta Astron. 73, 164 (2012).

3. D. Sziroczak and H. Smith, Prog. Aerospace Sci. 84, 1 (2016).

4. P. Gruhn and A. Gülhan, J. Propul. Power 27, 718 (2011).

5. C. Jiang et al., AIAA J. 55, 1 (2017).

6. R. T. Voland, L. D. Huebner and C. R. McClinton, Acta Astron. 59, 181 (2006).

7. S. A. Berry, A. H. Auslender and A. D. Dilley, J. Spacecr. Rockets 38, 853 (2001).

8. P. Rodi, The osculating flow field method of waverider geometry generation, AIAA Paper 2005-511 (2005).

9. E. K. Oswatitsch, NACA TM, 1140 (1944).

10. E. Schülein, AIAA J. 44, 1732 (2006). 